\title{
Generalized Relative AG Divergence of Type S and Information Inequalities
}

\author{
M.A.K Baig ${ }^{1}$, Arif Habib ${ }^{1}$, Mohd Afzal Bhat ${ }^{2}$ \\ ${ }^{1,2,}$ Department Of Statistics University Of Kashmir Srinagar-190006 (India).
}

Abstract: Shannon inequalities are well known in information theory. In this paper, we have proposed some generalized inequalities in terms of relative $A G$ divergence of type $s$ and $\chi^{2}$ divergence. The result obtained in particular lead us to some known divergence measure.

Key words: Csiszar's $f$ - divergence, Relative Arithmetic Geometric divergence, $\chi^{2}$ divergence, AG divergence of type $s$, Triangular discrimination, Information inequalities.

Let

\section{Introduction}

$\Delta_{n}=\left\{P=\left(p_{1}, p_{2}, \ldots, p_{n}\right), p_{i}>0, \sum_{i=1}^{n} p_{i}=1\right\}, n \geq 2$, be the set of complete finite discrete probability distributions. There are many information and divergence measures given in the literature on information theory and statistics. Some of these are symmetric with respect to probability distributions, while others are not. Throughout this paper it is understood that the probability distributions $P, Q \in \Delta_{n}$.

Some divergence measures are as follows.

$\chi^{2}$-Divergence (Pearson [6]

$$
\chi^{2}(P / / Q)=\sum_{i=1}^{n} \frac{\left(p_{i}-q_{i}\right)^{2}}{q_{i}}=\sum_{i==^{2}}^{n} \frac{p_{i}{ }^{2}}{q_{i}}-1
$$

and

$$
\chi^{2}(Q / / P)=\sum_{i=1}^{n} \frac{\left(q_{i}-p_{i}\right)^{2}}{p_{i}}=\sum_{i=1}^{n} \frac{q_{i}{ }^{2}}{p_{i}}-1
$$

AMS subject classification: 94 A17, 94 A24, 26 D15

Relative Jensen-Shannon divergence (Sibson [8], Sgarro [7])

$$
F(P / / Q)=\sum_{i=1}^{n} p_{i} l_{n}\left(\frac{2 p_{i}}{p_{i}+q_{i}}\right)
$$

Relative Arithmetic Geometric Divergence (Taneja [9])

$$
G(P / / Q)=\sum_{i=1}^{n}\left(\frac{p_{i}+q_{i}}{2}\right) l_{n}\left(\frac{p_{i}+q_{i}}{2 p_{i}}\right)
$$

Relative J-divergence (Dragomir et al [5])

$$
D(P / / Q)=\sum_{i=1}^{n}\left(p_{i}-q_{i}\right) l_{n}\left(\frac{p_{i}+q_{i}}{2 q_{i}}\right)
$$

and

$$
D(Q / / P)=\sum_{i=1}^{n}\left(q_{i}-p_{i}\right) l_{n}\left(\frac{p_{i}+q_{i}}{2 p_{i}}\right)
$$

II. Relative JS and AG divergence of type s.

Let us consider the relative JS and AG divergence of type s. 


$$
\Omega_{s}(P / / Q)=\left\{\begin{array}{ll}
F G_{s}(P / / Q)=[s(s-1)]^{-1}\left[\sum_{i=1}^{n} p_{i}\left(\frac{p_{i}+q_{i}}{2 p_{i}}\right)^{s}-1\right], & s \neq 0,1 \\
F(P / / Q)=\sum_{i=1}^{n} p_{i} l_{n}\left(\frac{2 p_{i}}{p_{i}+q_{i}}\right), & s=0 \\
G(P / / Q)=\sum_{i=1}^{n}\left(\frac{p_{i}+q_{i}}{2}\right) l_{n}\left(\frac{p_{i}+q_{i}}{2 p_{i}}\right), & s=1
\end{array}\right\}
$$

We have the following particular cases of $\Omega_{s}(P / / Q)$.

(i) $\Omega_{-1}(P / / Q)=\frac{1}{4} \Delta(P / / Q)$

(ii) $\Omega_{0}(P / / Q)=F(P / / Q)$

(iii) $\Omega_{1}(P / / Q)=G(P / / Q)$

(iv) $\Omega_{2}(P / / Q)=\frac{1}{8} \chi^{2}(Q / / P)$.

The expression $\Delta(P / / Q)$ appearing in part (i) is the well known triangular discrimination, and is given by

$$
\Delta(P / / Q)=\sum_{i=1}^{n} \frac{\left(p_{i}-q_{i}\right)^{2}}{p_{i}+q_{i}}
$$

\section{Csiszar's f- divergence and information inequalities.}

In this section we present Csiszar's $\mathrm{f}$ - divergence and bounds on it in terms of measure (2.1).

Given a convex function $f:[0, \infty) \rightarrow \Re$. The $\mathrm{f}$-divergence measure introduced by Csiszar [1] is given by

$$
C_{f}(p, q)=\sum_{i=1}^{n} q_{i} f\left(\frac{p_{i}}{q_{i}}\right)
$$

where $p, q \in \mathfrak{R}_{+}^{n}$.

The following two theorems are due to Csiszar and Korner [2].

Theorem 3.1: (joint convexity).Let $f:[0, \infty) \rightarrow \mathfrak{R}$ be convex, then the $C_{f}(p, q)$ is jointly convex in $\mathrm{p}$ and q, where $p, q \in \mathfrak{R}_{+}^{n}$.

Theorem 3.2: (Jensen's inequality). Let $f:[0, \infty) \rightarrow \mathfrak{R}$ be a convex function. Then for any $p, q \in \mathfrak{R}_{+}^{n}$, with $P_{n}=\sum_{i=1}^{n} p_{i}>0, Q_{n}=\sum_{i=1}^{n} q_{i}>0$, we have the inequality

$$
C_{f}(p, q) \geq Q_{n} f\left(\frac{P_{n}}{Q_{n}}\right) .
$$

The equality sign holds iff

$$
\frac{p_{1}}{q_{1}}=\frac{p_{2}}{q_{2}}=\ldots=\frac{p_{n}}{q_{n}} .
$$

In particular, for all $P, Q \in \Delta_{n}$, we have

$$
C_{f}(P / / Q) \geq f(1)
$$

With equality iff $\mathrm{P}=\mathrm{Q}$.

In view of Theorems 3.1 and 3.2, we state the following results. 
Result 3.1: For all $P, Q \in \Delta_{n}$, we note that

(i) $\Omega_{s}(P / / Q) \geq 0$ for any $s \in \mathfrak{R}$, with equality iff $\mathrm{P}=\mathrm{Q}$.

(ii) $\Omega_{s}(P / / Q)$ A convex function of the pair of distributions $(P, Q) \in \Delta_{n} \times \Delta_{n}$ and for any $s \in \mathfrak{R}$

Proof: Take

$$
\phi_{s}(u)=\left\{\begin{array}{ll}
{[s(s-1)]^{-1}\left[u\left(\frac{u+1}{2 u}\right)^{s}-u-s\left(\frac{1-u}{2}\right)\right],} & s \neq 0,1 \\
\frac{1-u}{2}-u l_{n}\left(\frac{u+1}{2 u}\right), & s=0 \\
\frac{u-1}{2}+\left(\frac{u+1}{2}\right) l_{n}\left(\frac{u+1}{2 u}\right), & s=1
\end{array}\right\}
$$

for all $u>0$ in (3.1), we get

$$
C_{f}(P / / Q)=\Omega_{s}(P / / Q)=\left\{\begin{array}{ll}
F G_{s}(P / / Q), & s \neq 0,1 \\
F(P / / Q), & s=0 \\
G(P / / Q), & s=1
\end{array}\right\}
$$

Moreover,

$$
\phi_{s}^{\prime}(u)=\left\{\begin{array}{ll}
(s-1)^{-1}\left\{\frac{1}{s}\left[\left(\frac{u+1}{2 u}\right)^{s}-1\right]+\frac{1}{2}\left[1-\frac{1}{u}\left(\frac{u+1}{2 u}\right)^{s-1}\right]\right\}, & s \neq 0,1 \\
\frac{1-u}{2(1+u)}-l_{n}\left(\frac{u+1}{2 u}\right), & s=0 \\
\frac{1}{2}\left[1-u^{-1}+l_{n}\left(\frac{u+1}{2 u}\right)\right], & s=1
\end{array}\right\}
$$

and

$$
\phi_{s}^{\prime \prime}(u)=\left\{\begin{array}{ll}
\frac{1}{4 u^{3}}\left(\frac{u+1}{2 u}\right)^{s-2}, & s \neq 0,1 \\
\frac{1}{u(u+1)^{2}}, & s=0 \\
\frac{1}{2 u^{2}(u+1)} & s=1
\end{array}\right\}
$$

Thus we have $\phi_{s}^{\prime \prime}(u)>0$ for all $u>0$, and hence, $\phi_{s}(u)$ is a convex for all $u>0$. Also, we have $\phi_{s}(1)=0$. In view of theorem 3.1 and 3.2 we have the proof of parts (i) and (ii), respectively.

The following theorem is due to Dragomir $[3,4]$.

Theorem 3.3: Let $f: \mathfrak{R}_{+} \rightarrow \mathfrak{R}$ be a differentiable convex function. Then for all $p, q \in \mathfrak{R}_{+}^{n}$, we have the inequalities

and

$$
f^{\prime}(1)\left(P_{n}-Q_{n}\right) \leq C_{f}(p, q)-Q_{n} f(1) \leq C_{f^{\prime}}\left(\frac{p^{2}}{q}, p\right)-C_{f^{\prime}}(p, q)
$$

$$
\mathrm{O} \leq C_{f}(p, q)-Q_{n} f\left(\frac{P_{n}}{Q_{n}}\right) \leq C_{f^{\prime}}\left(\frac{p^{2}}{q}, p\right)-\frac{P_{n}}{Q_{n}} C_{f^{\prime}},(p, q)
$$

where $f^{\prime}: \mathfrak{R}_{+} \rightarrow \mathfrak{R}$ is the derivative of $\boldsymbol{f}$.

If $\mathrm{f}$ is strictly convex then the equalities in (3.5) and (3.6) hold iff $\mathrm{p}=\mathrm{q}$. We can also write

$$
\rho_{f}(p, q)=C_{f^{\prime}}\left(\frac{p^{2}}{q}, p\right)-C_{f^{\prime}}(p, q)=\sum_{i=1}^{n}\left(p_{i}-q_{i}\right) f^{\prime}\left(\frac{p_{i}}{q_{i}}\right) .
$$

From the information theoretic point of view we shall use the following proposition.

Proposition 3.1: Let $f: \mathfrak{R}_{+} \rightarrow \mathfrak{K}$ be differentiable convex. If $P, Q \in \Delta_{n}$, then we can state 


$$
\mathrm{O} \leq C_{f}(P / / Q)-f(1) \leq C_{f^{\prime}}\left(\frac{P^{2}}{Q} / / P\right)-C_{f^{\prime}}(P / / Q)
$$

with equalities if $\mathrm{P}=\mathrm{Q}$.

In view of proposition 3.1, we have the following result.

Result 3.2: Let $P, Q \in \Delta_{n}$ and $s \in R$. Then

$$
0 \leq \Omega_{s}(P / / Q) \leq \eta_{s}(P / / Q)
$$

where

$$
\eta_{s}(P / / Q)=C_{\phi^{\prime} s}\left(\frac{P^{2}}{Q} / / P\right)-C_{\phi^{\prime} s}(P / / Q)
$$

$$
\eta_{s}(P / / Q)=\left\{\begin{array}{lr}
{[s(s-1)]^{-1} \sum_{i=1}^{n}\left(\frac{p_{i}-q_{i}}{p_{i}+q_{i}}\right)\left(\frac{p_{i}+q_{i}}{2 p_{i}}\right)^{s}\left[p_{i}+(1-s) q_{i}\right],} & s \neq 0,1 \\
D(Q / / P)-\frac{1}{2} \Delta(P / / Q), & s=0 \\
\frac{1}{2}\left[\chi^{2}(P / / Q)-D(Q / / P)\right], & s=1
\end{array}\right\}
$$

The proof is an immediate consequence of the proposition (3.1) by substituting $f($.$) by \phi_{s}($.$) ,$ where $\phi_{S}($.$) is given by (3.2).$

The measure (3.10) admits the following particular cases.

(i) $\eta_{0}(P / / Q)=D(Q / / P)-\frac{1}{2} \Delta(P / / Q)$

(ii) $\eta_{1}(P / / Q)=\frac{1}{2}\left[\chi^{2}(P / / Q)-D(Q / / P)\right]$

(iii) $\eta_{2}(P / / Q)=\frac{1}{8}\left[\chi^{2}(Q / / P)+\sum_{i=1}^{n}\left(\frac{p_{i}-q_{i}}{p_{i}}\right)^{2} q_{i}\right]$

We state the following corollaries as particular cases of result 3.2.

Corollary 3.1. We have

$$
\begin{aligned}
& 0 \leq F(P / / Q) \leq D(Q / / P)-\frac{1}{2} \Delta(P / / Q) \\
& 0 \leq G(P / / Q) \leq \frac{1}{2}\left[\chi^{2}(P / / Q)-D(Q / / P)\right] \\
& 0 \leq \frac{1}{8} \chi^{2}(Q / / P) \leq \eta_{2}(P / / Q) .
\end{aligned}
$$

Proof: (3.11) follows by taking $\mathrm{s}=0$, (3.12) follows by taking $\mathrm{s}=1$, (3.13) follows by taking $\mathrm{s}=2$ in (3.9).

Theorem 3.4: Let $f: I \subset \mathfrak{R}_{+} \rightarrow \mathfrak{R}$ be a mapping which is normalized, i.e., $f(1)=0$ and satisfy the assumptions:

(i) $\mathrm{f}$ is twice differentiable on ( $\mathrm{r}, \mathrm{R})$, where $0 \leq r \leq 1 \leq R<\infty$;

(ii) there exists the real constants $\mathrm{m}, \mathrm{M}$ such that $\mathrm{m}<\mathrm{M}$ and

$$
m \leq 4 x^{3}\left(\frac{x+1}{2 x}\right)^{2-s} f^{\prime \prime}(x) \leq M ; \quad \forall x \in(r, R), \quad s \in R
$$

If $P, Q \in \Delta_{n}$ are discrete probability distributions satisfying the assumption 


$$
\begin{gathered}
0<r \leq \frac{p_{i}}{q_{i}} \leq R<\infty, \text { then we have the inequalities } \\
m \Omega_{s}(P / / Q) \leq C_{f}(P / / Q) \leq M \Omega_{s}(P / / Q)
\end{gathered}
$$

and

$$
\begin{aligned}
m\left(\eta_{s}(P / / Q)-\right. & \left.\Omega_{s}(P / / Q)\right) \\
& =P_{f}(P / / Q)-C_{f}(P / / Q) \\
& \leq M\left(\eta_{s}(P / / Q)-\Omega_{s}(P / / Q)\right)
\end{aligned}
$$

where $\Omega_{s}(P / / Q), \rho_{f}(P / / Q)$ and $\eta_{s}(P / / Q)$ are as given by (2.1), (3.7) and (3.10) respectively.

Proof: Let us consider the functions $F_{m, s}($.$) and F_{M, s}($.$) given by$

$$
F_{m, s}(u)=f(u)-m \phi_{s}(u)
$$

and

$$
F_{M, s}(u)=M \phi_{s}(u)-f(u)
$$

respectively, where m, M are as given by (3.14) and the function $\phi_{s}($.$) is as given by (3.2).$

Since $f(u)$ and $\phi_{s}(u)$ are normalized, then $F_{m, s}($.$) and F_{M, s}($.$) are also normalized, i.e.,$ $F_{m, s}(1)=0$ and $F_{M, s}(1)=0$.Moreover, the functions $f(u)$ and $\phi_{s}(u)$ are twice differentiable.

Then in view of (3.4), we have

$$
\begin{aligned}
& F_{m, s}^{\prime \prime}(u)=f^{\prime \prime}(u)-m \phi_{s}^{\prime \prime}(u) \\
& F_{m, s}^{\prime \prime}(u)=f^{\prime \prime}(u)-m \frac{1}{4 u^{3}}\left(\frac{u+1}{2 u}\right)^{s-2} \\
& F_{m, s}^{\prime \prime}(u)=\frac{1}{4 u^{3}}\left(\frac{u+1}{2 u}\right)^{s-2}\left(4 u^{3}\left(\frac{u+1}{2 u}\right)^{2-s} f^{\prime \prime}(u)-m\right) \geq 0
\end{aligned}
$$

and

$$
\begin{aligned}
& F_{M, s}^{\prime \prime}(u)=M \phi_{s}^{\prime \prime}(u)-f^{\prime \prime}(u) \\
& F_{M, s}^{\prime \prime}(u)=M \frac{1}{4 u^{3}}\left(\frac{u+1}{2 u}\right)^{s-2}-f^{\prime \prime}(u) \\
& F_{M, s}^{\prime \prime}(u)=\frac{1}{4 u^{3}}\left(\frac{u+1}{2 u}\right)^{s-2}\left(M-4 u^{3}\left(\frac{u+1}{2 u}\right)^{2-s} f^{\prime \prime}(u)\right) \geq 0
\end{aligned}
$$

for all $u \in(r, R)$ and $s \in R$. Then the functions $F_{m, s}($.$) and F_{M, s}($.$) are convex on (r, R)$.

According to proposition 3.1, we have

$$
C_{F_{m, s}}(P / / Q)=C_{f}(P / / Q)-m \Omega_{s}(P / / Q) \geq 0
$$

and

$$
\text { (3.20) } \quad C_{F_{M, s}}(P / / Q)=M \Omega_{s}(P / / Q)-C_{f}(P / / Q) \geq 0
$$

Combining (3.19) and (3.20), we have

$$
m \Omega_{s}(P / / Q) \leq C_{f}(P / / Q) \leq M \Omega_{s}(P / / Q)
$$

We shall now prove the validity of inequalities (3.16). We have seen above that real mappings $F_{m, s}($.$) and F_{M, s}($.$) defined over R_{+}$given by (3.17) and (3.18), respectively are normalized, twice differentiable and convex related to $(r, R)$.

Applying the r.h.s of the inequalities (3.8), we have 


$$
C_{F_{m, s}}(P / / Q) \leq C_{F_{m, s}^{\prime}}\left(\frac{P^{2}}{Q} / / P\right)-C_{F_{m, s}^{\prime}}(P / / Q)
$$

and

$$
C_{F_{M, s}}(P / / Q) \leq C_{F_{M, s}^{\prime}}\left(\frac{P^{2}}{Q} / / P\right)-C_{F_{M, s}^{\prime}}(P / / Q)
$$

respectively.

Moreover,

$$
C_{F_{m, s}}(P / / Q)=C_{f}(P / / Q)-m \Omega_{s}(P / / Q)
$$

and

$$
C_{F_{M, s}}(P / / Q)=M \Omega_{s}(P / / Q)-C_{f}(P / / Q)
$$

In view of (3.21) and (3.23), we have

$$
C_{f}(P / / Q)-m \Omega_{s}(P / / Q) \leq C_{f^{\prime}-m \phi_{s}^{\prime}}\left(\frac{P^{2}}{Q} / / P\right)-C_{f^{\prime}-m \phi_{s}^{\prime}}(P / / Q)
$$

Thus,

$$
\begin{aligned}
C_{f}(P / / Q)- & m \Omega_{s}(P / / Q) \\
\leq & C_{f^{\prime}}\left(\frac{P^{2}}{Q} / / P\right)-m C_{\phi_{s}^{\prime}}\left(\frac{P^{2}}{Q} / / P\right)-C_{f^{\prime}}(P / / Q)+m C_{\phi_{s}^{\prime}}(P / / Q)
\end{aligned}
$$

Equivalently,

$$
\begin{aligned}
m\left[C_{\phi_{s}^{\prime}}\left(\frac{P^{2}}{Q} / / P\right)-C_{\phi_{s}^{\prime}}\right. & \left.(P / / Q)-\Omega_{s}(P / / Q)\right] \\
& \leq C_{f^{\prime}}\left(\frac{P^{2}}{Q} / / P\right)-C_{f^{\prime}}(P / / Q)-C_{f}(P / / Q)
\end{aligned}
$$

This gives,

$$
m\left[\eta_{s}(P / / Q)-\Omega_{s}(P / / Q)\right] \leq \rho_{f}(P / / Q)-C_{f}(P / / Q)
$$

Thus, we have 1.h.s of inequalities (3.16).

Again in view of (3.22) and (3.24), we have

$$
M \Omega_{s}(P / / Q)-C_{f}(P / / Q) \leq C_{m \phi_{s}^{\prime}-f^{\prime}}\left(\frac{P^{2}}{Q} / / P\right)-C_{m \phi_{s}^{\prime}-f^{\prime}}(P / / Q)
$$

Thus,

$$
\begin{aligned}
M \Omega_{s}(P / / Q) & -C_{f}(P / / Q) \\
& \leq M C_{\phi_{s}^{\prime}}\left(\frac{P^{2}}{Q} / / P\right)-C_{f^{\prime}}\left(\frac{P^{2}}{Q} / / P\right)-M C_{\phi_{s}^{\prime}}(P / / Q)+C_{f^{\prime}}(P / / Q)
\end{aligned}
$$

This gives,

Finally,

$$
\begin{aligned}
C_{f^{\prime}}\left(\frac{P^{2}}{Q} / / P\right)-C_{f^{\prime}}(P / / Q)-C_{f}(P / / Q) \\
\leq M\left[C_{\phi_{s}^{\prime}}\left(\frac{P^{2}}{Q} / / P\right)-C_{\phi_{s}^{\prime}}(P / / Q)-\Omega_{s}(P / / Q)\right]
\end{aligned}
$$

$$
\rho_{f}(P / / Q)-C_{f}(P / / Q) \leq M\left[\eta_{s}(P / / Q)-\Omega_{s}(P / / Q)\right]
$$

Thus we have r.h.s of the inequalities (3.16).

\section{Information inequalities}

In this section, we present particular cases of theorem 3.4.

\subsection{Information bounds in terms of relative Arithmetic Geometric divergence.}

In theorem (3.4) substituting $\mathrm{s}=1$, we have the following proposition

Proposition 4.1: Let $f: I \subset \mathfrak{R}_{+} \rightarrow \mathfrak{R}$ be a mapping which is normalized, i.e., $f(1)=0$ and satisfies the assumption: 
(i) $\mathrm{f}$ is twice differentiable on $(r, R)$, where $0 \leq r \leq 1 \leq R<\infty$;

(ii) there exists the real constants $\mathrm{m}, \mathrm{M}$ such that $\mathrm{m}<\mathrm{M}$ and

$$
m \leq 4 x^{3}\left(\frac{x+1}{2 x}\right) f^{\prime \prime}(x) \leq M \quad, \forall x \in(r, R) \quad, \quad s \in R \quad .
$$

If $P, Q \in \Delta_{n}$ are discrete probability distributions satisfying the assumption $0<r \leq \frac{p_{i}}{q_{i}} \leq R<\infty$, then we have the inequalities

$$
m G(P / / Q) \leq C_{f}(P / / Q) \leq M G(P / / Q)
$$

and

$$
\begin{aligned}
& m\left(\frac{1}{2} \chi^{2}(P / / Q)-\frac{1}{2} D(Q / / P)-\right.G(P / / Q)) \\
& \leq \rho_{f}(P / / Q)-C_{f}(P / / Q) \\
& \leq M\left(\frac{1}{2} \chi^{2}(P / / Q)-\frac{1}{2} D(Q / / P)-G(P / / Q)\right)
\end{aligned}
$$

where $G(P / / Q), \chi^{2}(P / / Q), D(Q / / P)$ and $\rho_{f}(P / / Q)$ are as given by (1.4), (1.1), (1.6), and (3.7) respectively.

Result 4.1: Let $P, Q \in \Delta_{n}$ and $s \in \mathfrak{R}$. Let there exists $\mathrm{r}, \mathrm{R}$ such that $\mathrm{r}<\mathrm{R}$ and $0<r \leq \frac{p_{i}}{q_{i}} \leq R<\infty$; $\forall i \in\{1,2, \ldots, n\}$, then the proposition 4.1 yields

$$
\begin{gathered}
\left(\frac{r+1}{2 r}\right)^{s-1} G(P / / Q) \leq \Omega_{s}(P / / Q) \leq\left(\frac{R+1}{2 R}\right)^{s-1} G(P / / Q) \quad, s \leq 1 \\
\left(\frac{R+1}{2 R}\right)^{s-1} G(P / / Q) \leq \Omega_{s}(P / / Q) \leq\left(\frac{r+1}{2 r}\right)^{s-1} G(P / / Q) \quad ; s \geq 1 \\
\left(\frac{r+1}{2 r}\right)^{s-1}\left[\frac{1}{2} \chi^{2}(P / / Q)-\frac{1}{2} D(Q / / P)-G(P / / Q)\right] \\
\leq \eta_{s}(P / / Q)-\Omega_{s}(P / / Q) \\
\leq\left(\frac{R+1}{2 R}\right)^{s-1}\left[\frac{1}{2} \chi^{2}(P / / Q)-\frac{1}{2} D(Q / / P)-G(P / / Q)\right] ; s \leq 1
\end{gathered}
$$

$$
\begin{aligned}
\left(\frac{R+1}{2 R}\right)^{s-1}\left[\frac{1}{2} \chi^{2}(P / / Q)\right. & \left.-\frac{1}{2} D(Q / / P)-G(P / / Q)\right] \\
& \leq \eta_{s}(P / / Q)-\Omega_{s}(P / / Q) \\
& \leq\left(\frac{r+1}{2 r}\right)^{s-1}\left[\frac{1}{2} \chi^{2}(P / / Q)-\frac{1}{2} D(Q / / P)-G(P / / Q)\right] ; s \geq 1
\end{aligned}
$$

Proof: Let us consider $f(u)=\phi_{s}(u)$, where $\phi_{s}(u)$ is given by (3.2).

According to expression (3.4), we have

$$
\phi_{s}^{\prime \prime}(u)=\frac{1}{4 u^{3}}\left(\frac{u+1}{2 u}\right)^{s-2}
$$

Let us define the function $g:[r, R] \rightarrow \mathfrak{R}$ such that

$$
g(u)=4 u^{3}\left(\frac{u+1}{2 u}\right) \phi_{s}^{\prime \prime}(u)
$$




$$
g(u)=\left(\frac{u+1}{2 u}\right)^{s-1}
$$

Then

$$
\sup _{u \in[r, R]} g(u)=\left\{\begin{array}{ll}
\left(\frac{R+1}{2 R}\right)^{s-1} & ; s \leq 1 \\
\left(\frac{r+1}{2 r}\right)^{s-1} & ; s \geq 1
\end{array}\right\}
$$

and

$$
\inf _{u \in[r, R]} g(u)=\left\{\begin{array}{ll}
\left(\frac{r+1}{2 r}\right)^{s-1} & ; s \leq 1 \\
\left(\frac{R+1}{2 R}\right)^{s-1} & ; s \geq 1
\end{array}\right\}
$$

where $\mathrm{r}$ and $\mathrm{R}$ are defined above.

Thus in view of (4.7), (4.8) and (4.1), we have inequalities (4.3) and (4.4). Again, in view of (4.7), (4.8) and (4.2), we have inequalities (4.5) and (4.6).

In view of result 4.2, we obtain the following corollary.

Corollary 4.1: under the conditions of result 4.1, we have

$$
\begin{aligned}
\left(\frac{2 r}{r+1}\right)\left[\frac{1}{2} \chi^{2}(P / / Q)-\right. & \left.\frac{1}{2} D(Q / / P)-G(P / / Q)\right] \\
& \leq D(Q / / P)-\frac{1}{2} \Delta(P / / Q)-F(P / / Q) \\
& \leq\left(\frac{2 R}{R+1}\right)\left[\frac{1}{2} \chi^{2}(P / / Q)-\frac{1}{2} D(Q / / P)-G(P / / Q)\right]
\end{aligned}
$$

$$
\begin{aligned}
\left(\frac{R+1}{2 R}\right)\left[\frac{1}{2} \chi^{2}(P / / Q)\right. & \left.-\frac{1}{2} D(Q / / P)-G(P / / Q)\right] \\
& \leq \eta_{2}(P / / Q)-\frac{1}{8} \chi^{2}(Q / / P) \\
& \leq\left(\frac{r+1}{2 r}\right)\left(\frac{1}{2} \chi^{2}(P / / Q)-\frac{1}{2} D(Q / / P)-G(P / / Q)\right)
\end{aligned}
$$

Proof: (4.9) follows by taking $s=-1$, (4.10) follows by taking $s=0$, in (4.3), (4.11) follows by taking $s=2$ in (4.4). (4.12) follows by taking $s=0$ in (4.5). (4.13) follows by taking $s=2$ in (4.6). While for $s=1$ we have equality sign.

\subsection{Information bounds in terms of $\chi^{2}$ divergence.}

In theorem (3.4) substituting $s=2$, we have the following proposition

Proposition 4.2: Let $f: I \subset \mathfrak{R}_{+} \rightarrow \mathfrak{R}$ be a mapping which is normalized, i.e., $f(1)=0$ and satisfies the assumptions

(i) $\mathrm{f}$ is twice differentiable on $(r, R)$, where $0 \leq r \leq 1 \leq R<\infty$;

(ii) there exists the real constants $\mathrm{m}, \mathrm{M}$ such that $\mathrm{m}<\mathrm{M}$ and 


$$
m \leq 4 x^{3} f^{\prime \prime}(x) \leq M, \quad \forall x \in(r, R),
$$

If $P, Q \in \Delta_{n}$ are discrete probability distributions satisfying the assumption

$$
0<r \leq \frac{p_{i}}{q_{i}} \leq R<\infty \text {, then we have the inequalities }
$$

and

$$
\frac{m}{8} \chi^{2}(Q / / P) \leq C_{f}(P / / Q) \leq \frac{M}{8} \chi^{2}(Q / / P)
$$

$$
m\left(\eta_{2}(P / / Q)-\frac{1}{8} \chi^{2}(Q / / P)\right) \leq \rho_{f}(P / / Q)-C_{f}(P / / Q) \leq M\left(\eta_{2}(P / / Q)-\frac{1}{8} \chi^{2}(Q / / P)\right)
$$

where $\chi^{2}(P / / Q)$ and $\rho_{f}(P / / Q)$ are as given by (1.2), (3.7) respectively.

$$
\text { Also } \eta_{2}(P / / Q)=\frac{1}{8}\left[\chi^{2}(Q / / P)+\sum_{i=1}^{n}\left(\frac{p_{i}-q_{i}}{p_{i}}\right)^{2} q_{i}\right] \text {. }
$$

Result 4.2: Let $P, Q \in \Delta_{n} \quad$ and. $s \in \mathfrak{R} \quad$ Let $\quad$ there $\quad$ exists $\quad \mathrm{r}, \quad \mathrm{R} \quad$ such $\quad$ that $\quad \mathrm{r}<\mathrm{R} \quad$ and $0<r \leq \frac{p_{i}}{q_{i}} \leq R<\infty ; \quad \forall i \in\{1,2, \ldots, n\}$, then the proposition 4.2 yields

$$
\begin{gathered}
\left(\frac{r+1}{2 r}\right)^{s-2}\left[\eta_{2}(P / / Q)-\frac{1}{8} \chi^{2}(Q / / P)\right] \\
\leq \eta_{s}(P / / Q)-\Omega_{s}(P / / Q) \\
\leq\left(\frac{R+1}{2 R}\right)^{s-2}\left[\eta_{2}(P / / Q)-\frac{1}{8} \chi^{2}(Q / / P)\right] ; s \leq 2 \\
\left(\begin{array}{r}
\left(\frac{R+1}{2 R}\right)^{s-2}\left[\eta_{2}(P / / Q)-\frac{1}{8} \chi^{2}(Q / / P)\right] \\
\leq \eta_{s}(P / / Q)-\Omega_{s}(P / / Q) \\
\quad \leq\left(\frac{r+1}{2 r}\right)^{s-2}\left[\eta_{2}(P / / Q)-\frac{1}{8} \chi^{2}(Q / / P)\right] ; s \geq 2
\end{array}\right.
\end{gathered}
$$$$
\frac{1}{8}\left(\frac{r+1}{2 r}\right)^{s-2} \chi^{2}(Q / / P) \leq \Omega_{s}(P / / Q) \leq \frac{1}{8}\left(\frac{R+1}{2 R}\right)^{s-2} \chi^{2}(Q / / P) ; s \leq 2
$$$$
\frac{1}{8}\left(\frac{R+1}{2 R}\right)^{s-2} \chi^{2}(Q / / P) \leq \Omega_{s}(P / / Q) \leq \frac{1}{8}\left(\frac{r+1}{2 r}\right)^{s-2} \chi^{2}(Q / / P) ; s \geq 2
$$

Proof: Let us consider $f(u)=\phi_{s}(u)$, where $\phi_{s}(u)$ is given by (3.2).

According to expression (3.4), we have

$$
\phi_{s}^{\prime \prime}(u)=\frac{1}{4 u^{3}}\left(\frac{u+1}{2 u}\right)^{s-2} .
$$

Let us define the function $g:[r, R] \rightarrow \mathfrak{R}$ such that

$$
\begin{aligned}
& g(u)=4 u^{3} \phi_{s}^{\prime \prime}(u) \\
& g(u)=\left(\frac{u+1}{2 u}\right)^{s-2}
\end{aligned}
$$

Then 


$$
\sup _{u \in[r, R]} g(u)=\left\{\begin{array}{ll}
\left(\frac{R+1}{2 R}\right)^{s-2} & ; s \leq 2 \\
\left(\frac{r+1}{2 r}\right)^{s-2} & ; s \geq 2
\end{array}\right\}
$$

and

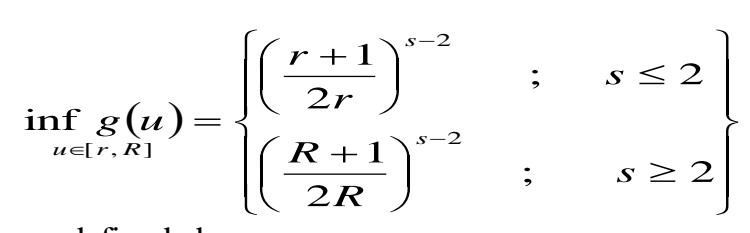

where $\mathrm{r}$ and $\mathrm{R}$, are defined above.

Thus in view of (4.20), (4.21) and (4.14), we have inequalities (4.16) and (4.17).

Again in view of (4.20), (4.21) and (4.15), we have inequalities (4.18) and (4.19).

In view of result 4.2 , we obtain the following corollaries.

Corollary 4.2: under the conditions of result 4.2, we have

$$
\begin{aligned}
\left(\frac{2 r}{r+1}\right)^{2}\left[\eta_{2}(P / / Q)-\frac{1}{8}\right. & \left.\mathcal{X}^{2}(Q / / P)\right] \\
& \leq D(Q / / P)-\frac{1}{2} \Delta(P / / Q)-F(P / / Q) \\
& \leq\left(\frac{2 R}{R+1}\right)^{2}\left[\eta_{2}(P / / Q)-\frac{1}{8} \mathcal{X}^{2}(Q / / P)\right]
\end{aligned}
$$

$$
\begin{aligned}
& \frac{1}{8}\left(\frac{2 r}{r+1}\right)^{3} \chi^{2}(Q / / P) \leq \frac{1}{4} \Delta(P / / Q) \leq \frac{1}{8}\left(\frac{2 R}{R+1}\right)^{3} \chi^{2}(Q / / P) \\
& \frac{1}{8}\left(\frac{2 r}{r+1}\right)^{2} x^{2}(Q / / P) \leq F(P / / Q) \leq \frac{1}{8}\left(\frac{2 R}{R+1}\right)^{2} \chi^{2}(Q / / P) \\
& \frac{1}{8}\left(\frac{2 r}{r+1}\right) x^{2}(Q / / P) \leq G(P / / Q) \leq \frac{1}{8}\left(\frac{2 R}{R+1}\right) \chi^{2}(Q / / P)
\end{aligned}
$$

$$
\begin{aligned}
\left(\frac{2 r}{r+1}\right)\left[\eta_{2}(P / / Q)-\frac{1}{8} \chi^{2}(Q / / P)\right] & \\
\leq & \frac{1}{2}\left[\chi^{2}(P / / Q)-D(Q / / P)\right]-G(P / / Q) \\
& \leq\left(\frac{2 R}{R+1}\right)\left[\eta_{2}(P / / Q)-\frac{1}{8} \chi^{2}(Q / / P)\right]
\end{aligned}
$$

Proof: (4.22) follows by taking s=-1, (4.23) follows by taking $s=0$, (4.24) follows by taking $s=1$ in (4.16). (4.25) follows by taking $\mathrm{s}=1$ and (4.26) follows by taking $\mathrm{s}=2$ in (4.18). While for $\mathrm{s}=2$, we have equality sign.

\section{References}

[1] I.Csiszar, Information type measures of differences of probability distribution and indirect observations, Studia Math. Hungarica 2(1967) 299-318.

[2] I.Csiszar, J.korner, Information theory: Coding theorems for discrete memoryless systems, Academic press, New York, 1981.

[3] S.S Dragomir, Some inequalities for the Csiszar $f$-divergence in information theory. Available from <http://rgmia.vu.edu.au/monographs/Csiszar.htm>

[4] S.S Dragomir, A Converse inequality for the Csiszar $\phi$-divergence, Inequality for Csiszar $\mathrm{f}$ divergence in information theory.Available from http://rgmia.vu.edu.au/monographs/Csiszar.htm

[5] S.S Dragomir, v. Gluscevic and C.E Pearce, Approximations for the Csiszar f- divergence via Mid points inequalities, In inequality theory and applications-Volume 1, Y.J Cho, J.K Kim and S.S Dragomir (Eds), Nova science publishers, Inc. Huntington, New york, 2001, pp 139-154.

[6] K.Pearson, On the criterion that a given system of deviations from the probable in the case of correlated system of variables is such that it can be reasonable supposed to have arisen from random sampling, Phil. Mag., 50(1900), 157-172.

[7] A.Sgarro, Information divergence and the dissimilarity of probability distribution, Estratto da calcolo, vol. XVII (3) (1981), 293-302.

[8] R. Sibson, Information radius, Z. wahrs.und verw Geb., (14) (1969), 149-160.

[9] I.J Taneja, Relative divergence measures and information inequalities-To appear: inequality theory and applications, volume 4, Y.J Cho, J. K Kim and S. S Dragomir(Eds), Nova Science publishers, Inc. Huntington, New york, 2004. 